\title{
Cloud Storage Protection Scheme Based on Fully Homomorphic Encryption
}

\author{
Mohammed A. Mohammed ${ }^{1}$ and Fadhil S. Abed ${ }^{2}$ \\ ${ }^{1}$ Department of Computer Science, College of Science, University of Sulaimani, \\ Sulaymaniyah, Kurdistan Region - F.R. Iraq \\ ${ }^{2}$ Department of Information Technology, Kalar Technical Institute, Sulaimani Polytechnic University, \\ Khanaqeen, Kurdistan Region - F.R. Iraq
}

\begin{abstract}
Cloud computing allows enterprises and individuals to have a less physical infrastructure of software and hardware. Nevertheless, there are some concerns regarding privacy protection which may turn out to be a strong barrier. Traditional encryption schemes have been used to encrypt the data before sending them to the cloud. However, the private key has to be provided to the server before any calculations on the data. To solve this security problem, this paper proposes a fully homomorphic encryption scheme for securing cloud data at rest. The scheme is based on prime modular operation, its security depends on factoring multiple large prime numbers $\left(p_{1}, p_{2}, \ldots p_{n}\right)$ up to $\mathrm{n}$, which is formed from very large prime numbers up to hundreds of digits as this is an open problem in mathematics. In addition, the elements of the secret key are derived from a series of mathematical operations and the calculation of an Euler coefficient within the modular of integers. Furthermore, it adds the complexity of noise to the plaintext using the number of users of the Cloud Service Provider. Moreover, its randomness is evaluated by the National Institute of Standards and Technology statistical tests, and the results demonstrating that the best statistical performance was obtained with this algorithm.
\end{abstract}

Index Terms - Cloud Computing Security, Cryptography, Fully Homomorphic Encryption, Information Security.

\section{INTRODUCTION}

Cloud computing plays an important role in storing and processing huge amounts of data since the fast progress of computer networks and big data (Hashem, et al. 2015). It provides flexible and on-demand remote storage and computing capabilities to its users. Nevertheless, as Gonzales et al. (2017) stated that cloud computing is not fully trustable since its users do not have full control over their data. Privacy protection and data leakage are the main risks for individuals and enterprises when it comes to migrating

ARO-The Scientific Journal of Koya University Vol. VIII, No.2 (2020), Article ID: ARO.10590, 08 pages DOI:10.14500/aro.10590

Received: 14 November 2019; Accepted: 16 November 2020 口ir.

Regular research paper: Published: 06 December 2020

Corresponding authơs e-mail:mohammed.anwar@univsul.edu.iq

Copyright (C) 2020 Mohammed A. Mohammed and Fadhil S. Abed.

This is an open-access article distributed undethe Creative Commons Attribution License. their data to cloud storage. The encryption techniques that require encrypted data on the cloud to be decrypted before performing any computation is still portend the privacy of stored data. Whereas, in Homomorphic Encryption operations can be performed directly on encrypted data without decrypting it. In addition, the result of the operation on encrypted data is equivalent to the result of its corresponding plaintext operation. This paper attempts to add an extra value to the privacy protection of cloud's data through proposing a new FHE scheme based on prime modular operation, which security depends on factoring multiple large prime numbers $\left(p_{1}, p_{2}, \ldots, p_{n}\right)$ up to $n$, which is formed from very large prime numbers up to hundreds of digits as this is an open problem in mathematics. Moreover, the randomness of the proposed work is evaluated by the well-known National Institute of Standards and Technology (NIST) test suite, which is widely used as a standard battery of tests to test randomness. The results of the proposed algorithm in the NIST statistical tests show that it produces the best statistical performance through passing all the tests.

\section{Problem Statement}

Nowadays, individuals and enterprises are seeking to access their private information anytime and anywhere. This leads them to deploy it onto cloud storage. However, they will be facing an extra amount of risks, which makes it challenging to maintain the security of outsourced data such as confidentiality, integrity, authentication, and privacy. For example, the hacking attack on PlayStation network in 2011 led it to leak millions of user accounts' passwords, physical addresses, credit card information, and other personal information. Later, the company stated that they could have encrypted the data on their network (Sangani, 2011). In addition, as reported by the Identity Theft Resource Center on May 31, 2018, thousands of FedEx customer records were exposed due to an unsecured server; some of the documents were passports, driving licenses, and security IDs (CyberScout, 2018). Therefore, Cloud Service Providers (CSPs) are required to keep an encrypted version of user's information on their storage. There is a variety of different techniques used for data encryption. Nevertheless, as the 
data resides on the cloud storage, it required to be decrypted before performing any operation on the data. This might cause privacy and confidentiality problems to the stored data. Whereas, homomorphic encryption allows performing computations on the encrypted data without decrypting it. Thus, HE solves the problems of confidentiality and privacy of the stored data inside the cloud. Therefore, this paper presents a new FHE scheme based on multiple large prime modular operation which is formed from very large prime numbers up to hundreds of digits. Hence, it makes the secret key very complicated which is difficult to retrieve it and resistance to different types of attacks.

\section{LITERATURE REVIEW}

Rivest et al. (1978) were proposed the first homomorphic encryption scheme and were partially homomorphic encryption (PHE). Then, Yao (1982) was also presented a PHE scheme. After that, RSA which was a multiplicative homomorphism introduced by Rivest et al. (1983). Afterward, several authors such as Goldwasser and Micali (1984), Elgamal (1985), and Paillier (1999) were also presented their PHE scheme. Subsequently, a fully homomorphic encryption (FHE) scheme suggested by Gentry (2009), which allows calculating of any number of addition and multiplication, hence compute arbitrary functions of encrypted data. Nevertheless, the scheme was based on Somewhat Homomorphic Encryption (SWHE), which increases the length and noise of ciphertext when calculation performs on the ciphertext. Consequently, van Dijk et al. (2010) have introduced FHE scheme that used elementary modular arithmetic and used Gentry's techniques to convert SWHE cryptosystem to FHE scheme. In addition, Smart and Vercauteren (2010) have presented an improved version of Smart-Vercauteren encryption scheme, the scheme was allowed several times decrease the ciphertext and keys lengths. In addition, IBM has released a software package named HElib in 2013, the company has implemented HE with further optimizations (Cheon, et al. 2019). Moreover, a HE scheme which is security dependent on the hardness of large integer factorization has been proposed by Xiao et al. (2012). Afterward, homomorphic encryption scheme has been worked on and improved by numerous authors, they have also tested it in a cloud computing system. Alattas and Elleithy (2013) have presented the application of algebraic homomorphic encryption mechanism and it was aiming at enhancing its security. In addition, several HE schemes such as RSA, Paillier, El-Gamal, and Gentry have been examined on a cloud computing environment by Tebaa and El Hajii (2014). In addition, Hayward and Chiang (2015) have improved Gentry's encryption in parallel processing and they have tested it in a private cloud domain. Furthermore, structured and simplified definitions in the homomorphic encryption discipline have been proposed by Armknecht et al. (2015). Moreover, SAM which is an FHE scheme over integers has been implemented by Shihab and Makki (2018). Furthermore, Li et al. (2016) constructed an efficient symmetric FHE scheme and utilized it to design a privacy-preserving-outsourced association rule mining scheme. Their proposal allows multiple data owners to jointly mine some association rules without sacrificing data privacy. The security of the HE scheme against the knownplaintext attacks was established by examining the difficulty of solving nonlinear systems. However, Wang et al. (2018) illustrated that the security of $\mathrm{Li}$ et al.'s HE is overvalued. They presented the retrieval and the second part can also be retrieved using a Euclidean algorithm to address the GCD problem of the first part of the secret key. Whereas, in 2019 ( $\mathrm{Li}$ et al.) used a lookup table to propose a protocol to evaluate any function using FHE.

Moreover, Ji and Shieh (2019) presented ways to reduce the computation complexity of encrypted data by adopting the concept of aggregate plaintext and proposing an efficient scheme to handle the comparison and swap operation, which is commonly used for sorting and searching in cloud computing. In late 2019, the authors of Jubrin et al. introduced FHE as an antidote to the challenges of security and privacy of cloud data computation; they also provided insight into future research directions in the field of FHE. Furthermore, Mohammed and Abed (2019) proposed an improved FHE based on N-primes, where the proposed model's security depends on the problem of factorization the integers to their primary numbers. Mert et al. (2020) presented two hardware architectures optimized for accelerating the encryption and decryption operations of the BFV/HE scheme with highperformance polynomial multipliers. In addition, in 2020, Tan et al. presented a private comparison algorithm on encrypted integers using FHE, which scales efficiently for the length of input integers, applying techniques from finite field theory. Whereas, Mohammed and Abed (2020) proposed a novel framework and an algorithm for securing cloud data at rest. The proposed framework guarantees users' privacy protection as they are communicating with an intermediary rather than with the cloud server directly.

Despite all the works presented previously, the randomness and robustness of the secret keys remain an open problem in the area of FHE. Therefore, this paper presents a new algorithm in which the elements of the secret key are derived from a series of mathematical operations and the calculation of an Euler coefficient within the modular of integers. Furthermore, it adds the complexity of noise to the plaintext by using the number of users of the CSP. Moreover, the proposed algorithm's randomness tests prove the best statistical performance was obtained with this algorithm. Furthermore, the algorithm works on encrypting and decrypting different languages such as Kurdish, English, and Arabic.

\section{HOMOMORPHIC ENCRYPTION}

In this section, HE scheme and its categories will be presented. Homomorphic encryption is divided into different categories, which are SWHE, FHE, and PHE. An encryption scheme is said to be homomorphic over an operation "+" if it supports the following equation, where $\mathrm{ms}$ is the plaintext message given to the encryption algorithm $E$ :

$$
E\left(m s_{1}\right)+E\left(m s_{2}\right)=E\left(m s_{1}+m s_{2}\right), \forall m s_{1}, m s_{2} \in M
$$




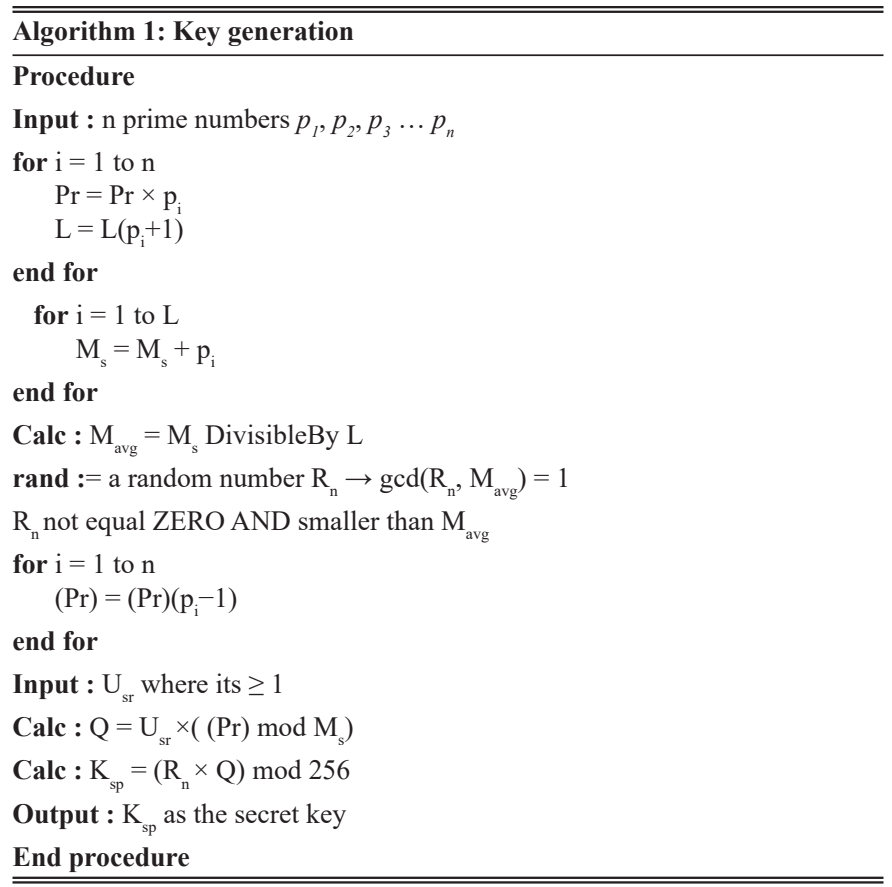

Somewhat homomorphic encryption allows addition and multiplication operations, however, both operations can be performed in a limited number Fellows and Koblitz (1994) and BNG by (Boneh-Goh-Nissim) (Dan, et al. 2005). Whereas, PHE allows one type of operation, either addition or multiplication, that is, Paillier, Goldwasser-Micali, Benaloh, El-Gamal, and RSA. On the other hand, FHE allows an unlimited number of both addition and multiplication on the ciphertext. It can be considered as ring homomorphism. As in mathematics, a ring is a set $\mathrm{R}$ equipped with two operations, "+" and " $\times "$ satisfying the eight axioms, known as the ring axioms. Examples of FHEs are FHE schemes Over Integers (dos Santos, et al. 2015), Simple FHE scheme (Li, et al. 2012), LWE-based FHE schemes (Regev, 2005), ideal latticebased FHE schemes (Gentry, 2009), and NTRU-like FHE schemes (Hoffstein, et al. 1998). Fig. 1 presents the popular schemes proposed after the Gentry's discovery.

\section{The Proposed Scheme}

The proposed scheme works on converting each plaintext character into its corresponding Unicode and then encrypts the derived Unicode by passing it to the encryption algorithm. In addition, the scheme also works on encrypting plaintexts in several languages such as Kurdish, English, and Arabic languages. In addition, the algorithm uses two different noises $r$ as the first noise is added to make the ciphertext more digestive, whereas the counter $i$ works on converting repeated characters in the text into different ciphertext values. The detailed notations used in the key generation, encryption, and decryption algorithms are presented in Table I. Subsequently, the working flow of the algorithms is illustrated in pseudocode.

Generating the Secret Key $K_{s p}$

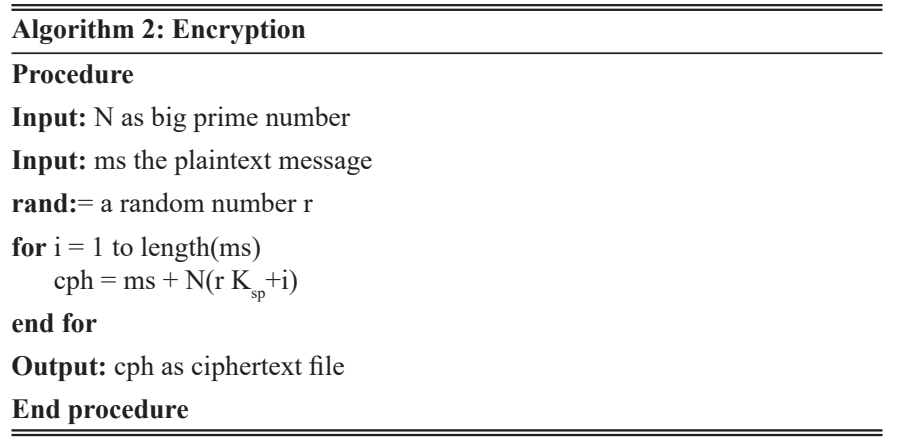

\begin{tabular}{l}
\hline \hline Algorithm 3: Decryption \\
\hline Procedure \\
for $\mathrm{i}=1$ to length(cph) \\
$\quad \mathrm{ms}=\mathrm{cph}$ mod $\mathrm{N}$ \\
end for \\
Output: ms as plaintext file \\
End procedure \\
\hline \hline
\end{tabular}

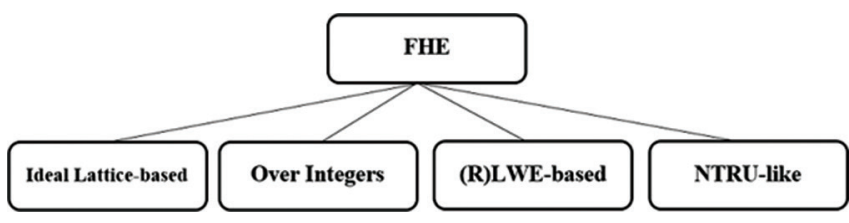

Fig. 1. Main FHE schemes after Gentry's discovery (Acar, et al. 2018).

At first choose multiple $P$ prime numbers $p_{1}, p_{2}, p_{3} \ldots p_{n}$ as secret keys, then calculate $P$ as $P=p_{1} \times p_{2} \times \ldots \times p_{n}$, calculate $L$ as $L=\left(p_{1}+1\right)\left(p_{2}+1\right) \ldots\left(p_{n}+1\right)$, then calculate $M_{s}=\sum_{i=1}^{m} F_{i}$, where $\mathrm{F}_{\mathrm{i}}=$ set of prime numbers up to $L$, and then calculate the average value of sum of all prime numbers as $M_{a v g}=\frac{M_{S}}{L}$, then choose a random number $R_{n}$ that satisfies $\operatorname{gcd}\left(R_{n}, M_{\text {avg }}\right)=1$, $1<R_{n}<M_{\text {avg }}$, then select $U_{s r}$ as it is the number of existing users of the cloud system $\left\{U_{s r 1}, U_{s r 2}, \ldots, U_{s r n}\right\}$, where $U_{s r} \geq 1$, calculate $\theta(P)=\left(p_{1}-1\right) \ldots\left(p_{n}-1\right), \quad$ and calculate $Q=U_{s r} \times\left(\theta(P) \bmod M_{s}\right)$ and finally calculate $K_{s p}$ as:

$$
K_{s p}=\left(R_{n} \times Q\right) \bmod 256
$$

Mod 255 is taken as this is because the secret key values are derived from a series of mathematical calculations that are within a certain scale between 1 and 255 so that the values resulting from the equation are not very large and prevent any slowness in the calculation process.

\section{Encryption algorithm}

$$
c p h=m s+N\left(r K_{s p}+i\right)
$$

Decryption algorithm

$$
\operatorname{ms}=c p h \bmod N
$$


TABLE I

List OF NOTATIONS

\begin{tabular}{ll}
\hline \hline Notations & Details \\
\hline$c p h$ & Ciphertext \\
$m s$ & The plaintext message \\
$r$ & Noise added to the ciphertext \\
$N$ & Big prime integer \\
$K_{s p}$ & Secret key \\
$i$ & Counter added as extra noise to the ciphertext works on \\
& converting repeated character into different ciphertext value. \\
$p_{l}, p_{2}, p_{3} \ldots p_{n}$ & Multiple prime numbers \\
$P$ & Is the multiplications of prime numbers \\
$L$ & Is the multiplication of each prime number plus one \\
$M_{\mathrm{s}}$ & The summation of prime numbers up to $L$ \\
$M_{\text {avg }}$ & Average of all prime numbers \\
$R_{n}$ & Is a random number where gcd $\left(\mathrm{R}_{\mathrm{n}}, \mathrm{M}_{\text {avg }}\right)=1$ \\
$U_{s r}$ & Is the number of CSP's users \\
\hline \hline CSP: Cloud service provider
\end{tabular}

\section{A. Proof of Homomorphism}

This subsection will illustrate the homomorphism of the proposed scheme, assume there are two ciphertexts $\mathrm{cph}_{1}$ and $\mathrm{cph}_{2}$ where $c p h_{1}=m s_{1}+N\left(r_{1} K_{\text {sup }}+i\right), \quad c p h_{2}=m s_{2}+N\left(r_{2} K_{\text {sup }}+i\right)$, and $c p h \bmod N \equiv m s$, where $m s<N$, otherwise, we must take $(\operatorname{ms} \bmod N)$.

\section{Homomorphism (Addition)}

Assume that the sum of two ciphertexts $c p h_{1}$ and $c p h_{2}$ is denoted by $\quad\left(c p h^{+}=c p h_{1}+c p h_{2}\right)$ so $c p h^{+}=c p h_{1}+c p h_{2}=\left(m s_{1}+m s_{2}\right)+N\left(r_{1} K_{s p}+i\right)+N\left(r_{2} K_{s p}+i\right)$, nonetheless $N\left(r_{1} K_{s p}+i\right)+N\left(r_{2} K_{s p}+i\right)=N K_{s p}\left[\begin{array}{l}\left(r_{1}+r_{2}\right) \\ +2 i\end{array}\right]=0$ Then $m s^{+}=\left(c p h_{1}+c p h_{2}\right) \quad \bmod \quad N=m s_{1}+m s_{2}$

\section{Homomorphism (Multiplication)}

Assume that the sum of two ciphertexts $c p h_{1}$ and $c p h_{2}$ is denoted by $\left(c p h^{*}=c p h_{1} * c p h_{2}\right) c p h^{*}=\left[m s_{1}+N\left(r_{1} K_{s p}+i\right)\right] \times$ $\left[m s_{2}+N\left(r_{2} K_{s p}+i\right)\right] c p h^{*}=\left[\mathrm{ms}_{1} \times \mathrm{ms}_{2}+\mathrm{ms}_{1} \times N\left(r_{2} K_{s p}+i\right)\right]$ $\left.+N\left(r_{1} K_{s p}+i\right) \times m s_{2}+N\left(r_{1} K_{s p}+i\right) \times N\left(r_{2} K_{s p}+i\right)\right]$ Then $N \times$ $\left[m s_{1} \times N\left(r_{2} K_{s p}+i\right)+\left(r_{1} K_{s p}+i\right) \times m s_{2} N\left(r_{1} K_{s p}+i\right) \times N\left(r_{2} K_{s p}\right.\right.$ $+i) \bmod N]=0$ So that, $c p h^{*}=m s_{1} \times m s_{2}+0=m s_{1} \times m s_{2}$.

\section{RESUlt AND ANALYSIS}

In this section, the results gained from the proposed scheme will be presented through numerous tests on English, Kurdish, and Arabic languages. To test the proposed scheme, it is implemented with Java programming language and processed on a computer with the following features: Intel Core i7 processor, HDD hard drive, 16 GB RAM, and Windows 10 64-bit. At first, the generation of the secret key is illustrated then it will be used for all the tests presented in this section.

Secret key generation

Choose a set of prime numbers for as $p_{1}=31, p_{2}=59$ and $p_{3}=73 \quad$ then $\theta(P)=30 \times 58 \times 72=125280$, $P=31 \times 59 \times 73=133517 . \quad$ Then, calculate
$L=(31+1)(59+1)(73+1)=142080$, so $M_{s}=129548351731$,

$M_{\text {avg }}=911798$, assume $U_{s r}=35$ and $R_{n}=15$ then

$Q=4384800$, finally $K_{s p}=224$.

\section{A. Test on English Language}

The proposed algorithm will be tested on an English language text of "Hello world; this is a new Fully Homomorphic algorithm." For this test, the secret key will be $K_{s p}=224$ as generated previously, and $N=524287$ which is

big prime number, then choose a random number as $r=62598$ the ciphertext of the given text after applying the proposed algorithm on it will be:

$7351527148296 \quad 73515276726127351528196906$

$7351528721193 \quad 73515292454837351529769691$

735153029406573515308183447351531342634

735153186691573515323911947351532915413

735153343978473515339640597351534488347

735153501264473515355368487351536061208

735153658550573515371097097351537634061

$73515381582837351538682648 \quad 7351539206926$

735153973123173515402554317351540779756

735154130409073515418283687351542352655

735154287695573515434011537351543925480

$7351544449806 \quad 73515449740917351545498380$

735154602266573515465469547351547071244

735154759552973515481198087351548644096

735154916837773515496925977351550216949

735155074124773515512655297351551789824

735155231411473515528383927351553362690

73515538869657351554411257

Table II and Fig. 2 illustrate the performance of the proposed algorithm tested on different file sizes that contain plaintext written in the English language.

\section{B. Test on Kurdish Language}

This test illustrates the proposed algorithm tested on a

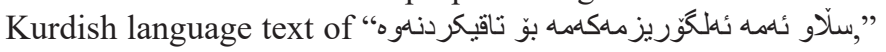
and the same values used for testing English language text and the ciphertext will be:

$\begin{array}{llllllllllllll}7351527149811 & 7 & 3 & 5 & 1 & 5 & 2 & 7 & 6 & 7 & 4 & 2 & 2 & 8\end{array}$ 735152819837373515287226937351529245404 $73515297712337351530295695 \quad 7351530819838$ 735153134426973515318668397351532392668 $7351532917130 \quad 73515334412727351533965666$ 735153448997673515350141147351535538556 735153606268973515365869957351537111426 735153763566973515381600007351538684143 735153920857473515397311447351540256975 735154078142073515413040057351541829838 735154235412273515428784367351543402861 735154392711373515444512807351544975565 735154549987573515460243057351546548451 7351547072879

Table III and Fig. 3 illustrate the performance of the proposed algorithm tested on different file sizes that contain plaintext written in the Kurdish language. 
TABLE II

Testing the Proposed Algorithm on Different File Sizes Written in THE ENGLISH LANGUAGE

\begin{tabular}{lcc}
\hline \hline File sizes & Encryption (ms) & Decryption (ms) \\
\hline $10 \mathrm{~KB}$ & 378 & 414 \\
$20 \mathrm{~KB}$ & 397 & 448 \\
$40 \mathrm{~KB}$ & 413 & 499 \\
$80 \mathrm{~KB}$ & 459 & 570 \\
$160 \mathrm{~KB}$ & 486 & 625 \\
$320 \mathrm{~KB}$ & 529 & 710 \\
$500 \mathrm{~KB}$ & 599 & 831 \\
$1 \mathrm{MB}$ & 748 & 1081 \\
$2 \mathrm{MB}$ & 981 & 1597 \\
$4 \mathrm{MB}$ & 1698 & 2703 \\
$8 \mathrm{MB}$ & 2531 & 4843 \\
$16 \mathrm{MB}$ & 4234 & 8432 \\
\hline \hline
\end{tabular}

TABLE III

Testing the Proposed Algorithm on Different File Sizes Written in THE KURDish LANGUAGE

\begin{tabular}{lcc}
\hline \hline File sizes & Encryption (ms) & Decryption $(\mathrm{ms})$ \\
\hline $10 \mathrm{~KB}$ & 375 & 406 \\
$20 \mathrm{~KB}$ & 391 & 438 \\
$40 \mathrm{~KB}$ & 421 & 500 \\
$80 \mathrm{~KB}$ & 437 & 562 \\
$160 \mathrm{~KB}$ & 485 & 688 \\
$320 \mathrm{~KB}$ & 578 & 766 \\
$500 \mathrm{~KB}$ & 594 & 814 \\
$1 \mathrm{MB}$ & 734 & 1109 \\
$2 \mathrm{MB}$ & 1031 & 1625 \\
$4 \mathrm{MB}$ & 1702 & 2609 \\
$8 \mathrm{MB}$ & 2848 & 4582 \\
$16 \mathrm{MB}$ & 5471 & 9018 \\
\hline \hline
\end{tabular}

\section{Test on Arabic Language}

This time the proposed algorithm will be tested on an Arabic text of "مرحبا نقام لكم خوارزمية جديدة," also the values from the first test will be used and the ciphertext is as follow:

$7351527149829 \quad 73515276740967351528198379$ 735152872266173515292469477351529769691 735153029555273515308198357351531344103 735153186841273515323911267351532916985 735153344127173515339655607351534488274 735153501411173515355384247351536062678 $73515365869757351537111263 \quad 7351537635569$ 735153815986173515386841157351539206857 735153973269273515402569827351540781296 73515413055567351541829837

The previous tests presented that the proposed algorithm can be performed on different languages, and it produces different cipher-values for all plaintext values and also for the repeated character within the same text. In addition, the rest of this section will present the performance of the proposed algorithm performed on different file sizes written in English, Kurdish, and Arabic languages. Table IV and Fig. 4 illustrate the performance of the proposed algorithm tested on different file sizes that contain plaintext written in the Arabic language.

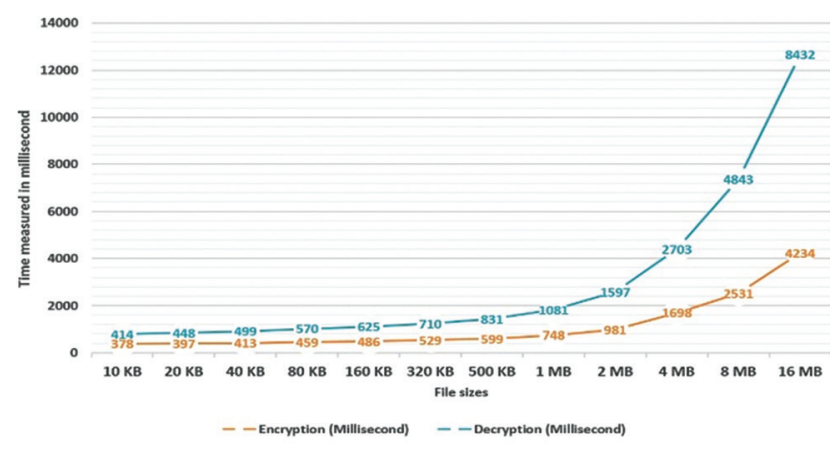

Fig. 2. Encryption and decryption time on English language plaintext file.

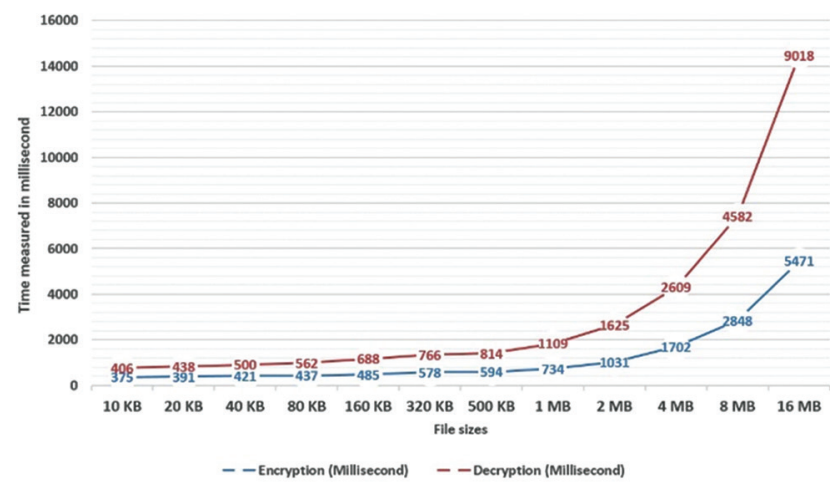

Fig. 3. Encryption and decryption time on Kurdish language plaintext file.

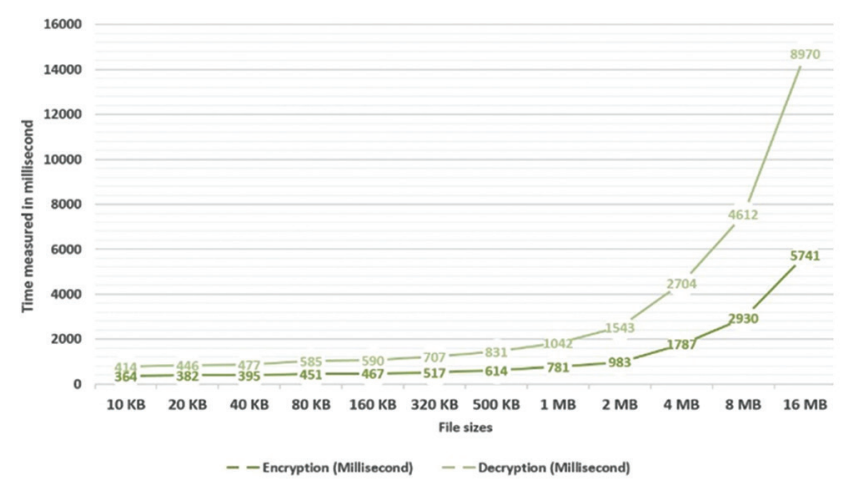

Fig. 4. Encryption and decryption time on Arabic language plaintext file.

The results of the previous tests show that the proposed algorithm is capable on encrypting plaintexts written in different languages efficiently regardless of the file size. In addition, it can be observed from the results that the algorithm performs almost the same performance on the same file sizes of various languages. Table $\mathrm{V}$ and Fig. 5 present a comparison of the previous tests gained from the proposed algorithm. As it is illustrated, the encryption and decryption time for all three languages are vary and almost works the same. Such as the encryption time of $20 \mathrm{~KB}$ Arabic text-file requires less time than the encryption time on its corresponding English and Kurdish text-file. Whereas, the encryption time of 2 MB English text-file takes less time than Kurdish and Arabic text-files. 


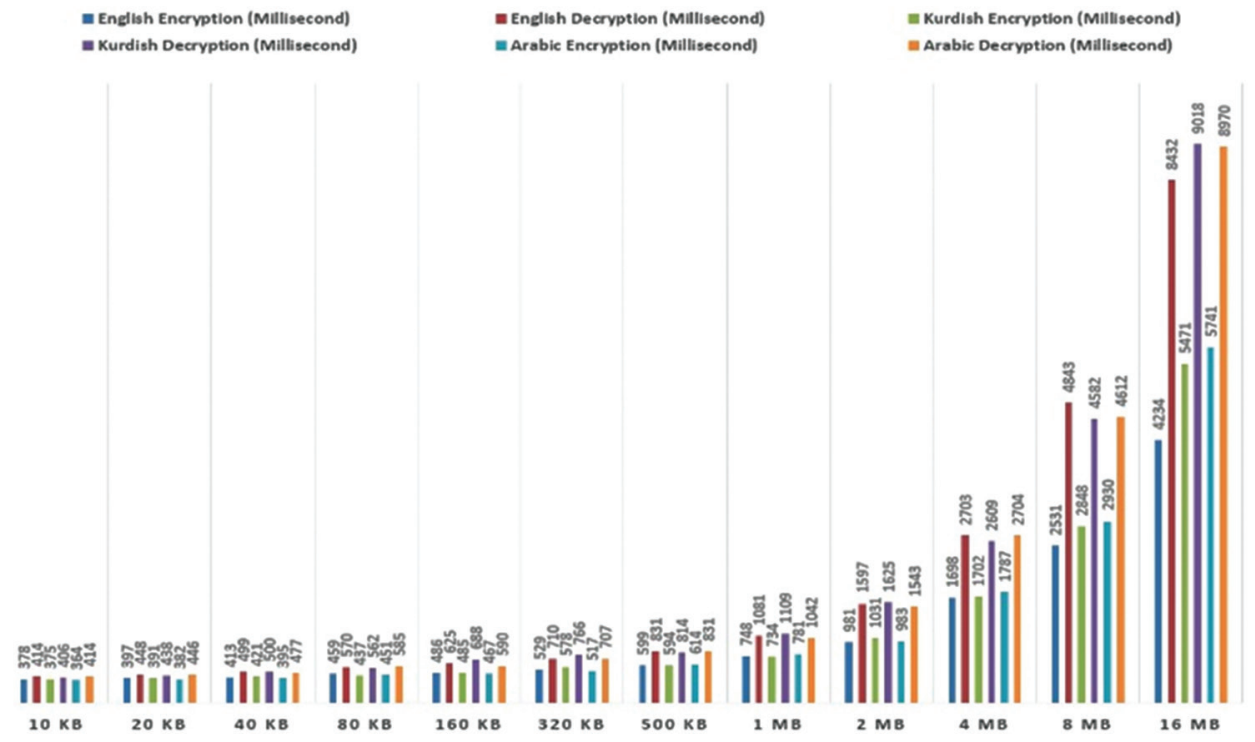

Fig. 5. Encryption and decryption time on English, Kurdish, and Arabic language plaintext file.

TABLE IV

Testing the Proposed Algorithm on Different File Sizes Written in the Arabic Language

\begin{tabular}{lcc}
\hline \hline File sizes & Encryption $(\mathrm{ms})$ & Decryption $(\mathrm{ms})$ \\
\hline $10 \mathrm{~KB}$ & 364 & 414 \\
$20 \mathrm{~KB}$ & 382 & 446 \\
$40 \mathrm{~KB}$ & 395 & 477 \\
$80 \mathrm{~KB}$ & 451 & 585 \\
$160 \mathrm{~KB}$ & 467 & 590 \\
$320 \mathrm{~KB}$ & 517 & 707 \\
$500 \mathrm{~KB}$ & 614 & 831 \\
$1 \mathrm{MB}$ & 781 & 1042 \\
$2 \mathrm{MB}$ & 983 & 1543 \\
$4 \mathrm{MB}$ & 1787 & 2704 \\
$8 \mathrm{MB}$ & 2930 & 4612 \\
$16 \mathrm{MB}$ & 5741 & 8970 \\
\hline \hline
\end{tabular}

TABLE V

Time CompleXity of the Basic Arithmetic Operations

\begin{tabular}{lcc}
\hline \hline Operation & $\begin{array}{c}\text { Time complexity } \\
\text { of binary integers } \\
\text { of size } \mathrm{n}\end{array}$ & $\begin{array}{c}\text { Time complexity } \\
\text { of decimal digits of } \\
\text { size } \mathrm{n}\end{array}$ \\
\hline Addition $\mathrm{x}+\mathrm{y}$ & $\mathrm{O}(\mathrm{n})$ & $\mathrm{O}(\log (\mathrm{n}))$ \\
Subtraction $\mathrm{x}-\mathrm{y}$ & $\mathrm{O}(\mathrm{n})$ & $\mathrm{O}(\log (\mathrm{n}))$ \\
Multiplication $\mathrm{x} \times \mathrm{y}$ & $\mathrm{O}\left(\mathrm{n}^{2}\right)$ & $\mathrm{O}\left(\left(\log \left(\mathrm{n}^{2}\right)\right)\right.$ \\
Division and Modular & $\mathrm{O}\left(\mathrm{n}^{2}\right)$ & $\mathrm{O}\left(\left(\log \left(\mathrm{n}^{2}\right)\right)\right.$ \\
Inverse $\mathrm{x}^{-1}$ & $\mathrm{O}\left(\mathrm{n}^{2} \log (\mathrm{n})\right)$ & $\left.\mathrm{O}(\log (\mathrm{n}))^{3}\right)$ \\
Modular exponentiation $\mathrm{x}^{\mathrm{n}}$ & $\mathrm{O}\left(\mathrm{n}^{2} \log (\mathrm{n})\right)$ & $\left.\mathrm{O}(\log (\mathrm{n}))^{3}\right)$ \\
\hline \hline
\end{tabular}

\section{Big O Notation (Time Complexity)}

Searching for the "best" in algorithms is the main concern of algorithms' designers, and this can be achieved through using O-notation. The aim of studying the time complexity of an algorithm is to determine whether the algorithms' running time is $O(f(N))$ for some function $f($ ) or not. Table VI illustrates the complexity of the basic arithmetic operations in $Z_{n}$ (Sagheer, 2012).
The input numbers of encryption and decryption algorithms should be analyzed at first before performing any calculation of the time complexity. The input numbers are either binary integers or decimal digits, whereas the time complexity of the first mentioned is $O(n)$ and the time complexity of decimal digits is $O(\log (n))$, this excluding constant number whose complexity is $O(1)$. Since, $\mathrm{n}$ is the size of input numbers.

1) Time complexity of DGHV scheme

Let $n$ be the size of input message unit.

Encryption function:

$c p h=m s+2 r+p \times q$

Then: $T(c p h)=O(n)+T(2 r)+O\left(n^{2}\right)$

$T(2 r)=O(n)$, by shift operation

$T(c p h)=O(2 n)+O\left(n^{2}\right) \equiv O\left(n^{2}\right)$ bit operation.

Decryption function:

$m s=(c p h \bmod p) \bmod 2$

Then: $T(m s)=O\left(n^{2}\right)$ bit operation

2) Time complexity of SDC scheme

Let $n$ be the size of input message unit.

Encryption function:

$c p h=m s+p+r \times p \times q$

Then: $T(c p h)=O(n)+O(n)+O\left(2\left(n^{2}\right)\right)$

$T(c p h)=O(2(n))+O\left(2\left(n^{2}\right)\right) \equiv O\left(n^{2}\right)$ bit operation.

Decryption function:

$m s=c p h \bmod p$

Then: $T(m s)=O\left(n^{2}\right)$ bit operation.

3) Time complexity of the proposed algorithm Let $n$ be the size of input message, $n$ is decimal digit.

Encryption function:

$c p h=m s+N\left(r K_{s p}+i\right)$

Then: $T(c p h)=O(2(\log (n)))+O\left(2(\log (n))^{2}\right)$ 
TABLE VI

Comparing the Results of the Algorithm Gained from Encrypting and Decrypting English, Kurdish, and Arabic Language’s PlainteXts

\begin{tabular}{|c|c|c|c|c|c|c|}
\hline \multirow[t]{2}{*}{ File sizes } & \multicolumn{2}{|c|}{ English } & \multicolumn{2}{|c|}{ Kurdish } & \multicolumn{2}{|c|}{ Arabic } \\
\hline & Encryption (ms) & Decryption (ms) & Encryption (ms) & Encryption (ms) & Encryption (ms) & Encryption (ms) \\
\hline $10 \mathrm{~KB}$ & 378 & 414 & 375 & 406 & 364 & 414 \\
\hline $20 \mathrm{~KB}$ & 397 & 448 & 391 & 438 & 382 & 446 \\
\hline $40 \mathrm{~KB}$ & 413 & 499 & 421 & 500 & 395 & 477 \\
\hline $80 \mathrm{~KB}$ & 459 & 570 & 437 & 562 & 451 & 585 \\
\hline $160 \mathrm{~KB}$ & 486 & 625 & 485 & 688 & 467 & 590 \\
\hline $320 \mathrm{~KB}$ & 529 & 710 & 578 & 766 & 517 & 707 \\
\hline $500 \mathrm{~KB}$ & 599 & 831 & 594 & 814 & 614 & 831 \\
\hline $1 \mathrm{MB}$ & 748 & 1081 & 734 & 1109 & 781 & 1042 \\
\hline $2 \mathrm{MB}$ & 981 & 1597 & 1031 & 1625 & 983 & 1543 \\
\hline $4 \mathrm{MB}$ & 1698 & 2703 & 1702 & 2609 & 1787 & 2704 \\
\hline $8 \mathrm{MB}$ & 2531 & 4843 & 2848 & 4582 & 2930 & 4612 \\
\hline $16 \mathrm{MB}$ & 5741 & 8970 & 5471 & 9018 & 5741 & 8970 \\
\hline
\end{tabular}

\section{$T(c p h) \equiv O\left(\log (n)^{2}\right)$}

Decryption function:

$m s=c p h \bmod N$

Then: $T(m s)=O\left((\log (n))^{2}\right)$, Where, $\left(\log _{2} n\right)$ is the number of bits of $n$

\section{E. Resistance to Attacks}

In this section, the resistance of the proposed algorithm to different types of attacks such as Key Generation and Character Repetition, Brute Force Attack, and Mathematical Attack are illustrated.

4) Key generation and character repetition

The proposed algorithm encrypts each file with a different key, and it depends on a variable that is different for every cloud user. In addition, the algorithm encrypts the repetition of each character into different values. Thus, the attacker cannot analyze character repetition in the file. Consequently, the combination of different keys for each file and different values for the same character allows our proposed algorithm to provide a strong encryption method.

5) Brute force attack

In the proposed algorithm, the strength of large prime numbers depends on the multiplication of $n$ prime numbers $p_{p}$, $p_{2}, \ldots p_{n}$. Thus, it is difficult to break the large prime number into multiple primes as compared to the existing algorithms. Furthermore, the multiple prime numbers increase the level of difficulty to break the security of the algorithm. In addition, the use of the addition noises makes it more difficult to break.

6) Mathematical attack

This kind of attack occurs when the attacker determines the values of $p$ and $q$. In our proposed algorithm, it is reduced as the algorithm uses multiple numbers of primes, and it is hard to derive any of those primes from the multiplication result.

\section{F. Results of NIST Statistical Tests}

The randomness of this novel proposal is evaluated by the well-known NIST test suite. Table VII shows the test results of the proposed algorithm from the NIST statistical
TABLE VII

NIST SP 800-22 TEST RESULTS FOR THE NAZUZ ALGORITHM

\begin{tabular}{lcl}
\hline \hline Tests & $\boldsymbol{P}$-value & Result \\
\hline Frequency (Monobits) & 0.997743 & Success \\
Block frequency & 0.999936 & Success \\
Cumulative sums (Cusum) & 0.983782 & Success \\
Runs & 0.982544 & Success \\
Longest run of ones & 0.993900 & Success \\
Rank & 0.999594 & Success \\
Discrete Fourier transform & 0.074478 & Success \\
Non-overlapping template matching & 0.999975 & Success \\
Overlapping template matching & 0.856322 & Success \\
Universal statistical & 0.999620 & Success \\
Approximate entropy & 0.999961 & Success \\
Random excursions & 0.997529 & Success \\
Random excursions variant & 0.837424 & Success \\
Serial & 0.999995 & Success \\
Linear complexity & 0.999438 & Success \\
\hline \hline
\end{tabular}

tests, demonstrating that the best statistical performance was obtained with this algorithm.

\section{CONCLUSION}

It has been said that homomorphic encryption is the change point of cryptography, as it protects data regardless of its situation, whether the data are in transit or at rest. This helped CSPs to use this new technique for data protection. This paper is proposed a new FHE scheme based on prime modular operation. The scheme performs encryption and decryption on plaintext values regardless of the written language of the plaintext English, Kurdish, Arabic, or any other languages as well as special characters. In addition, the scheme encrypts repeated characters of the plaintext into different ciphertext values which increases the security of the ciphertext. The randomness of the proposal scheme is evaluated by the well-known NIST test suite (widely used as a standard battery of tests to test randomness). The results of the proposed algorithm in the NIST statistical tests show that it produces the best statistical performance through passing 
all the tests. Moreover, the proposed scheme demonstrates good security for the stored data on the cloud.

\section{REFERENCES}

Acar A, Aksu H., Uluagac A.S. and Conti, M., 2018. A survey on homomorphic encryption schemes: Theory and implementation. ACM Computing Surveys, 51(4), pp.1-35.

Alattas, R. and Elleithy, K., 2013. Cloud Computing Algebra Homomorphic Encryption Scheme Based on Fermat's Little Theorem. The American Society of Engineering Education, Northfield, VT, USA.

Armknecht, F., Boyd, C., Carr, C., Gjøsteen, K., Jaschke, A., Reuter, C. and Strand, M., 2015. A guide to fully homomorphicencryption. IACR Cryptology ePrint Archive, 2015, 1192.

Cheon, J., Choe, H., Lee, D. and Son, Y., 2019. Faster linear transformations in HElib, revisited. IEEE Access, 7, pp.50595-50604.

CyberScout., 2018. Data Breach Reports. Identity Theft Resource Center, Berkeley, CA, USA.

Dan, B., Eu-Jin, G. and Kobbi, N. 2005. Evaluating 2-DNF formulas on ciphertexts. In: Proceedings of Theory of Cryptography Conference. Vol. 3378. Springer, Berlin. pp.325-341.

dos Santos, L.C., Bilar, G.R. and Pereira, F.D., 2015. Implementation of the Fully Homomorphic Encryption Scheme Over Integers with Shorter Keys. In: $20157^{\text {th }}$ International Conference on New Technologies, Mobility and Security (NTMS), Paris, France.

Elgamal, T., 1985. A public key cryptosystem and a signature scheme based on discrete logarithms. IEEE Transactions on Information Theory, 31(4), pp.469-472.

Fellows, M. and Koblitz, N., 1994. Combinatorial cryptosystems galore! In: Finite Fields: Theory, Applications, and Algorithms. American Mathematical Society, Providence, Rhode Island. pp.51-61.

Gentry, C., 2009. A Fully Homomorphic Encryption Scheme, PhD. Stanford University, United States.

Gentry, C., 2009. Fully Homomorphic Encryption Using Ideal Lattices. In: Proceedings of the $41^{\text {st }}$ Annual ACM Symposium on Symposium on Theory of Computing STOC '09, Bethesda, Maryland, USA.

Goldwasser, S. and Micali, S., 1984. Probabilistic encryption. Journal of Computer and System Sciences, 28(2), pp.270-299.

Gonzales, D., Kaplan, J., Saltzman, E., Winkelman, Z. and Woods, D., 2017. Cloud-trust a security assessment model for infrastructure as a service (IaaS) clouds. IEEE Transactions on Cloud Computing, 5(3), pp.523-536.

Hashem, I., Yaqoob, I., Anuar, N., Mokhtar, S., Gani, A. and Khan, S.U., 2015. The rise of "big data" on cloud computing: Review and open research issues. Information Systems, 47, pp.98-115.

Hayward, R. and Chiang, C., 2015. Parallelizing fully homomorphic encryption for a cloud environment. Journal of Applied Research and Technology, 13(2), pp.245-252.

Hoffstein, J., Pipher, J. and Silverman, J., 1998. NTRU: A ring-based public key cryptosystem. In: Lecture Notes in Computer Science. Springer Science+Business Media, Berlin, Germany. pp.267-288.

Ji, J. and Shieh, M., 2019. Efficient comparison and swap on fully homomorphic encrypted data. In: 2019 IEEE International Symposium on Circuits and Systems (ISCAS), Sapporo, Japan, pp.1-4.

Jubrin, A.M., Izegbu, I. and Adebayo, O.S., 2019. Fully homomorphic encryption: An antidote to cloud data security and privacy concems. In: 2019 $15^{\text {th }}$ International Conference on Electronics, Computer and Computation (ICECCO), Abuja, Nigeria, pp.1-6.
Li, J., Song, D., Chen, S. and Lu, X., 2012. A Simple Fully Homomorphic Encryption Scheme Available in Cloud Computing. In: 2012 IEEE $2^{\text {nd }}$ International Conference on Cloud Computing and Intelligence Systems, Hangzhou, China.

Li, L., Lu, R., Choo, K.R., Datta A. and Shao J., 2016. Privacy-preservingoutsourced association rule mining on vertically partitioned databases. IEEE Transactions on Information Forensics and Security, 11(8), pp.1847-1861.

Li, R., Ishimaki, Y. and Yamana H., 2019. Fully homomorphic encryption with table lookup for privacy-preserving smart grid. In: 2019 IEEE International Conference on Smart Computing (SMARTCOMP), Washington, DC, USA, pp.19-24.

Mert, A.C., Öztürk E. and Savaş, E., 2020. Design and Implementation of Encryption/Decryption Architectures for BFV Homomorphic Encryption Scheme. Vol. 28. IEEE Transactions on Very Large Scale Integration Systems, pp.353-362.

Mohammed, M.A. and Abed, F.S., 2019. An improved fully homomorphic encryption model based on N-primes. Kurdistan Journal of Applied Research, 4(2), pp.40-49.

Mohammed, M.A. and Abed, F.S., 2020. A symmetric-based framework for securing cloud data at rest. Turkish Journal of Electrical Engineering and Computer Sciences, 28(1), pp.347-361.

Paillier, P., n.d. Public-key cryptosystems based on composite degree residuosity classes. Advances in Cryptology Eurocrypt, 99, pp.223-238.

Regev, O., 2005. On Lattices, Learning with Errors, Random Linear Codes, and Cryptography. In: Proceedings of the $37^{\text {th }}$ annual ACM Symposium on Theory of Computing STOC '05, Baltimore, Maryland, USA.

Rivest, R., Shamir, A. and Adleman, L., 1983. A method for obtaining digital signatures and public-key cryptosystems. Communications of the ACM, 26(1), pp.96-99.

Rivest, R.L.; Adleman, L. and Dertouzos, M.L., 1978. On data banks and privacy homomorphisms. In: Foundations of Secure Computation. Academia Press, Cambridge, Massachusetts. pp.169-179.

Sagheer, A.M., 2012. Elliptic Curves Cryptographic Techniques. In: $20126^{\text {th }}$ International Conference on Signal Processing and Communication Systems, Gold Coast, QLD, pp.1-7.

Sangani, K., 2011. Sony security laid bare. Engineering and Technology, 6(8), pp.74-77.

Shihab, H. and Makki, S., 2018. Design of fully homomorphic encryption by prime modular operation. Telfor Journal, 10(2), pp.118-122.

Smart, N. and Vercauteren, F., 2010. Fully homomorphic encryption with relatively small key and ciphertext sizes. Public Key Cryptography, 2010, pp.420-443.

Tan, B.H.M., Lee, H.T., Wang, H., Ren, S.Q. and Khin, A.M.M., 2020. Efficient private comparison queries over encrypted databases using fully homomorphic encryption with finite fields. IEEE Transactions on Dependable and Secure Computing, p.1.

Tebaa, M. and El Hajii, S., 2014. Secure cloud computing through homomorphic encryption. Computing Research Repository, 5, 1409.

van Dijk, M., Gentry, C., Halevi, S. and Vaikuntanathan, V., 2010. Fully homomorphic encryption over the integers. Advances in Cryptology Eurocrypt, 2010, pp.24-43.

Wang, B., Zhan, Y. and Zhang, Z., 2018. Cryptanalysis of a symmetric fully homomorphic encryption scheme. IEEE Transactions on Information Forensics and Security, 13(6), pp.1460-1467.

Xiao, L., Bastani, O. and Yen, I., 2012. An Efficient Homorphic Encryption Protocol for Multi-user Systems. IACR Cryptology ePrint Archive, Lyon, France.

Yao, A., 1982. Protocols for Secure Computations. In: $23^{\text {rd }}$ Annual Symposium on Foundations of Computer Science (sfcs 1982), Washington, DC. 